\title{
Bezafibrate improves mitochondrial fission and function in DNM1L deficient patient cells
}

Liza Douiev $^{1 *}$, Ruth Sheffer ${ }^{1}$, Gabriella Horvath ${ }^{2}$, Ann Saada $^{1,3 *}$

${ }^{1}$ Department of Genetic and Metabolic Diseases and Jacques Roboh Department of Genetic Research, Hadassah Medical Center, Jerusalem 9112001, Israel.

${ }^{2}$ Division of Biochemical Diseases, Department of Pediatrics, University of British Columbia, Vancouver, BC, Canada

${ }^{3}$ Faculty of Medicine, Hebrew University of Jerusalem, 9112001, Israel

*corresponding authors

Prof. Ann Saada (Reisch), Metabolic Laboratory, Department of Genetic and Metabolic Diseases Hadassah Medical Center, Jerusalem 9112001, Israel. annsr@hadassah.org.il, anns@ekmd.huji.ac.il

Liza Douiev, Metabolic Laboratory, Department of Genetic and Metabolic Diseases Hadassah Medical Center, Jerusalem 9112001, Israel liza.douiev@mail.huji.ac.il 


\begin{abstract}
Mitochondria are involved in many cellular processes and their main role is cellular energy production. They constantly undergo fission and fusion, and these counteracting processes are under strict balance. The cytosolic dynamin-related protein 1, Drp1 or dynamin-1-like protein (DNM1L) mediates mitochondrial and peroxisomal division. Defects in the DNM1L gene results in a complex neurodevelopmental disorder with heterogeneous symptoms affecting multiple organ systems. Currently there is no curative treatment available for this condition. We have previously described a patient with a de novo heterozygous c.1084G>A (p.G362S) DNM1L mutation and studied the effects of a small molecule, Bezafibrate, on mitochondrial functions in this patient's fibroblasts compared to controls. Bezafibrate normalized growth on glucose-free medium, ATP production, oxygen consumption and s improved mitochondrial morphology in patient's fibroblasts, albeit concomitantly causing a mild increase ROS production. Further studies would be needed to show the consistency of the response to Bezafibrate, possibly using fibroblasts from patients with different mutations in DNM1L, and this treatment should be confirmed in clinical trials. However, taking into account the favorable effects in our study, we suggest that Bezafibrate could be a possible treatment option for patients with certain DNM1L mutations.
\end{abstract}

\title{
Key Words
}

DNM1L, Drp1, mitochondrial disease, mitochondrial fission-fusion, Bezafibrate, fibroblast 


\section{Introduction}

Mitochondria are cytosolic double membrane-bound organelles that found in nucleated eukaryotic cells. They are involved in many cellular processes; including regulation of cell proliferation and differentiation, intracellular calcium homeostasis, regulation of cell death and etc. However their major role is cellular energy (ATP) production via oxidative phosphorylation (OXPHOS) [1]. Mitochondria are highly dynamic, organelles, constantly undergoing fission and fusion. Their number, shape and location undergo changes throughout the cell cycle and during different environmental conditions, and energy status. This process is mediated by a highly regulated balance between the counteracting processes of fission versus fusion [2-7].

Symptoms of mitochondrial disorders affecting OXPHOS are heterogeneous, affecting most of the organ systems in the body and include muscle weakness, neurological deficits (including seizures), vision or hearing loss, poor growth and involvement of other organs such as gastrointestinal, cardiac or respiratory systems. These symptoms can present at any age, but if presenting early, usually imply a much more severe disease course, in many cases being fatal in infancy or early childhood [1] Defects in mitochondrial genes encoding proteins involved in mitochondrial dynamics proteins causing abnormal fission or fusion can also affect OXPHOS and result in clinical disease. Among these, mutations, in the gene encoding dynamin-1-like protein, DNM1L are increasingly associated with human diseases. Since the first patient with mutation in the DNM1L gene, presenting with lethal epileptic encephalopathy was described by Waterham and colleagues in 2007 [8], there have been several case reports published. As of today, there are over 18 patients described, most of them having heterozygous de novo variants in the Middle Domain, that were shown to have negative dominant effect, others have variants in the GTPase Head Domain or in the GTPase Head Domain. The clinical phenotypes vary between neonatal hypotonia, microcephaly, apnea, persistent elevation of lactate, focal status progressing into generalized status epilepticus with devastating neurological impairment and death early in infancy or childhood. Some other patients had a relative normal early development then developed severe epileptic encephalopathy with intractable seizures and regression after an intercurrent illness, infection or vaccination [9-20].

DNM1L is the human analogue to Drp1, a member of the dynamin superfamily of cytoplasmic GTPases, is an evolutionarily conserved protein which mediates mitochondrial and peroxisomal division, and is also involved in apoptotic regulation. Drp1 regulates mitochondrial 
fission through assembly of fission foci and distribution of mitochondrial tubules through the cytoplasm, which is essential for normal mitochondrial function and turnover. Drp1 contains three domains prototypical of the dynamin-like GTPase class, with an N-terminal GTPase head, a middle-domain (MD) and a C-terminal GTPase effector domain (GED), as well as a nonconserved Variable Domain (VD) Drp1 is recruited to the mitochondrial outer membrane upon fission triggering. It self-assembles into a fission complex that forms a ring-like structure that constricts the organelle, thus providing the force to produce a local fission event. The mechanism of initial recruitment of Drp1 to the outer mitochondrial membrane is a rate-limiting step of mitochondrial fission. A shift in the mitochondrial fusion and fission equilibrium can drive the mitochondria to elongate or fragment in response to certain cellular demands, suggesting an intricate relationship between mitochondrial morphology and function. Consequences of blockade of mitochondrial fission are: abnormal distribution of mitochondria ROS (reactive oxygen species) generation, accumulation of polyubiquinated mitochondria and loss of respiratory function[21-25, reviewed in 26-27].

Despite major advances in the scientific and medical community's ability to diagnose and identify the genetic causes for mitochondrial diseases, treatment remains a major challenge. Generally, treatment has been supportive and symptomatic with little effect on overall outcome of the disease. Several therapeutic approaches have been proposed and investigated over the past years. The administration of small molecule-based approach is one of them. The use of small molecules aims to improve mitochondrial functions such as respiratory efficiency and ATP production, reducing the levels of free radicals, etc. These small molecules are divided into several groups; including antioxidants, co-factors, mitochondrial biogenesis promoting factors, and more [reviewed in 28-31]. One such molecule is Bezafibrate, which is considered as a peroxisome proliferator-activated receptor alpha (PPAR $\alpha$ ) agonist. PPARs are ligand-activated transcription factors, which upon activation form heterodimers with the retinoid $\mathrm{X}$ receptor (RXR). This heterodimer binds to a specific recognition sequence that is located within a gene regulatory region of PPARs-target genes. PPAR family is composed of three isoforms; each has a variety of target genes and exhibits different tissue distributions. Some of their target genes are nuclear-encoded respiratory chain (RC) genes, and others are mitochondrial biogenesis and fatty oxidation genes. The molecular mechanisms underlying Bezafibrate effects on mitochondrial function and biogenesis are partially explained by its ability to upregulate the expression of nuclear-encoded mitochondrial genes [31, 32]. 
The main goal of the current study was to examine the effect of Bezafibrate on mitochondrial function in derived from a patient carrying a dominant negative DNM1L mutation. Our results suggest a possible therapeutic intervention for patients that carrying mutations in fission genes.

\section{Materials and Methods}

\subsection{Materials}

Cell culture media and solutions were obtained from Biological Industries (Kibbutz Beit Ha'emek, Israel). Fluorescent mitochondrial dyes were purchased from Invitrogen (Carlsbad, CA, USA), $\mathrm{H}_{2}$ DCFDA from Biotium (Hayward, CA, USA), and the ATPlite ${ }^{\mathrm{TM}}$ was from Perkin Elmer (Waltham, MA, USA). Other reagents were purchased from Sigma-Aldrich (Rehovot, Israel) at the highest purity available.

\subsection{Cell culture}

Previously established primary skin fibroblast cell lines available for the study were derived from the patient, harboring the de novo heterozygous c.1084G>A (p.G362S) mutation [10] and controls with informed consent and IRB \#0485-09 approval. Cells were cultured in a permissive high-glucose-DMEM medium containing $4.5 \mathrm{~g} / \mathrm{L}$ glucose supplemented with $15 \%$ fetal calf serum (FCS), $1 \%$ penicillin-streptomycin, $365 \mu \mathrm{g} / \mathrm{mL}$ L-glutamine, $110 \mu \mathrm{g} / \mathrm{mL}$ pyruvate, and $50 \mu \mathrm{g} / \mathrm{mL}$ uridine at $37^{\circ} \mathrm{C}, 5 \% \mathrm{CO}_{2}$. Cell growth was also evaluated in a restrictive glucose-free DMEM medium supplemented with $10 \%$ dialyzed FCS and $5 \mathrm{mM}$ galactose. To evaluate Bezafibrate replaced with a fresh medium with or without $100 \mu \mathrm{M}$ Bezafibrate $(200 \mathrm{mM}$ stock solution in DMSO, stored at $-20^{\circ} \mathrm{C}$ ) for 72 hours prior to assessments.

\subsection{Assays in microtiter wells}

Assays were performed in microtiter wells essentially as we have previously described [33-35], $3000 /$ well were seeded overnight and replaced with new permissive or restrictive medium, in the presence or absence of Bezafibrate. Cell content, ATP production, mitochondrial membrane potential (MMP) and reactive oxygen species (ROS) were evacuated as follows; Cell content was measured by a colorimetric method using methylene blue (MB), The results are presented as growth in glucose-free medium divided by average cellular growth in high-glucose medium. 
ATP production was measured using the ATPlite ${ }^{T M}$ luminescence assay system, in digitoninpermeabilized cells in the presence of glutamate and malate we have previously described [35]. Mitochondrial membrane potential (MMP) was estimated using double staining with MitoTracker Green and Tetramethylrhodamine ethyl ester (Molecular Probes, Eugene, OR, USA), and intracellular ROS production was measured in a fluorometric assay using 2',7'dichlorodihydrofluorescein diacetate ( $\mathrm{H}_{2} \mathrm{DCFDA}$ ) (Biotium, Harvard, CA, USA), as we previously described.

All values were normalized to cell growth as measured by $M B$ in parallel wells for each separate experiment. Results are presented as mean \pm SEM of experiments performed in triplicate wells on at least three separate occasions. Readings were obtained with a Synergy HT microplate reader instrument. Statistical analysis was made by student's t-test (IMB-SPSS 20). A p value of $<0.05$ was considered statistically significant.

\subsection{Microscopy}

Mitochondrial morphology was visualized using MitoTracker Red CM-H2XRos(MTR) . 3×10 fibroblasts were seeded on $35 \mathrm{~mm}$ glass bottom tissue culture plates in high-glucose medium and incubated with or without Bezafibrate at $37^{\circ} \mathrm{C}, 5 \% \mathrm{CO}_{2}$. Subsequently, the cells were incubated with MTR $(2 \mu \mathrm{M})$, for 30 minutes at $37^{\circ} \mathrm{C}, 5 \% \mathrm{CO}_{2}$ in the dark. The cells were visualized live by fluorescent, confocal microscopy (Olympus FV300) and the analysis and quantification were performed on at least 50 cells on two different occasions, using ImageJ software http://imagej.nih.gov/ij/

\subsection{Oxygen consumption}

Oxygen consumption rate (OCR) was measured using an XF24 extracellular flux analyzer (Seahorse Biosciences, North Billeric, MA, USA) as we have previously described [35] in the absence and presence of Bezafibrate in the medium $72 \mathrm{~h}$ prior to measurement. 


\section{Results}

\subsection{Bezafibrate normalizes growth, ATP production and oxygen consumption.}

We have previously reported functional OXPHOS deficits and aberrant mitochondrial morphology in the DNM1L patient's fibroblasts, thus we set out to evaluate the effect of Bezafibrate on several some OXPHOS parameters [10].To evaluate growth, patients and control fibroblasts were incubated high-glucose (permissive) and a glucose-free, galactose containing (restrictive), media. Notably, cells grown under permissive conditions derive most of their energy from glucose via glycolysis, whereas growth in the restrictive medium forces cells to rely on mitochondrial oxidation of fatty acids, glutamate via the OXPHOS. Accordingly, cells with OXPHOS defects exhibit defective growth on glucose-free [36].This was also in the present case, as growth ratio in glucose-free compared to high-glucose media was significantly lower in patient fibroblasts (0.62) compared to control fibroblasts (0.81) (Fig.1 A). The patient's fibroblasts supplemented with Bezafibrate showed significantly increased growth ratio (0.95). This was even a higher ratio than control cells while; the mild effect on these was not statistically significant. We verified our, preliminary results [10], confirming that, the patient's fibroblasts produced decreased levels (66\% of controls) of ATP (Fig.1 B). Supplementation with Bezafibrate induced a significant increase ( $170 \%$ relative to no additive), thereby restoring (114\% of controls) ATP production. Bezafibrate mildly increased in the ATP production also in controls albeit not statistically significant.

Although, previously we did not detect any significant decrease in MMP we still evaluated this parameter to evaluate the effect of Bezafibrate and observed a minor increase, which was not significantly different from the controls cells (Fig. 1C). Bezafibrate was not able to ameliorate the significantly elevated basal ROS production in patient cells (Fig.1D). Moreover, the supplementation slightly increased ROS production with $20 \%$ in the patient's cells but with no apparent effect on controls.

Maximal oxygen consumption in the patients' fibroblasts was decreased to $68 \%$ of controls and normalized (105\%) with Bezafibrate treatment. The ratio of basal to maximal oxygen consumption did not differ between patient and control, treated or untreated, thus there was no evidence of uncoupling. 

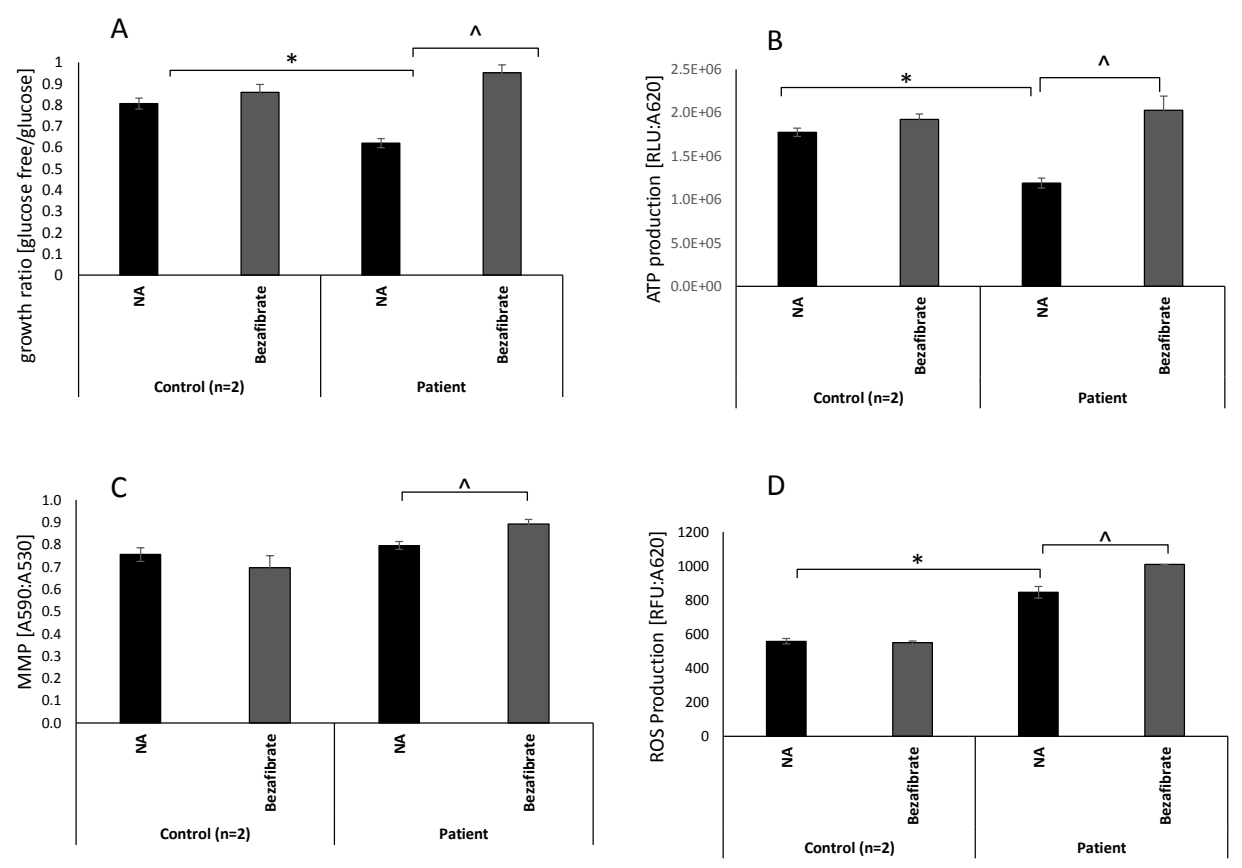

Figure 1. Patient and control fibroblasts' growth, ATP production, mitochondrial membrane potential and ROS production

Patient and controls fibroblasts were seeded on 96-well plates and incubated for 72 hours without additive (NA) or in the presence of Bezafibrate. Cell growth (A) was measured by MB and presented as the ratio between cell growth in glucose-free and growth in high-glucose. ATP production from glutamate +malate (B) measured was measured by luciferin-luciferase luminescence. MMP (C) was measured using double staining with MitoTracker Green (MTG) and Tetramethylrhodamine ethyl ester (TMRE).

Intracellular ROS production (D) was measured using fluorometry with $\mathrm{H}_{2}$ DCFDA. Values are presented as normalized mean $\pm \mathrm{SEM}$; ${ }^{*} p<0.05$ patient compared control in corresponding medium; ${ }^{\wedge} p<0.05$ Beza fibrate treated compared to NA. 


\section{Bezafibrate improves mitochondrial morphology}

As we have previously shown [10] mitochondrial morphology in patient fibroblasts is aberrant with markedly elongated mitochondria compared to normal fibroblasts grown under the same conditions (Fig.2 A-B). Evidently Bezafibrate had an apparent significant effect on mitochondrial morphology, which is mainly expressed by a less elongated phenotype in patient's fibroblasts while controls were largely unaffected (Fig.2 C- D). The histogram in Fig.2 E, depicts the quantitative estimation of average mitochondrial length, shows that patient's mitochondria are more than four times longer relative to controls. Bezafibrate markedly shortened mitochondrial length in the patient's cells to less than half of the untreated cells, although remaining more elongated that controls.

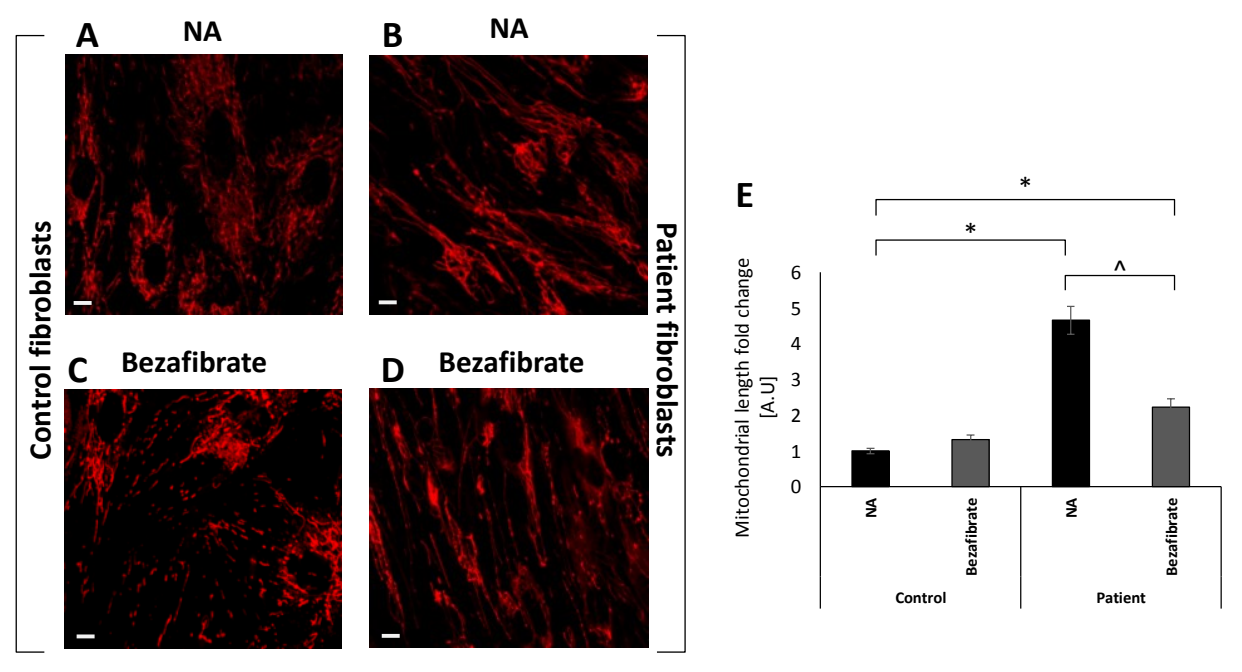

Figure 2. Mitochondrial morphology.

Control $(A, C)$ and patients fibroblasts $(B, D)$ were seeded in $35 \mathrm{~mm}$ glass bottom tissue culture plates. The following day, the medium was replaced with fresh high-glucose medium without $(A, B)$ additive (NA) or in the presence of Bezafibrate $(C, D)$ and incubated for 72 hours. Mitochondrial morphology was visualized by MitoTracker Red CM-H2XRos, Relative mitochondrial length was calculated and depicted ( E ) using ImageJ software. Mean normalized to control no additive \pm SEM; ${ }^{*} p<0.05$ compared to NA control $\wedge p<0.05$ compared to same cell with no additive. Scale bar $=10 \mu \mathrm{m}$ 


\section{Discussion}

The mitochondrion is a highly dynamic organelle that constantly fuses and divides. These two processes are crucial for proper mitochondrial function and mutation in components regulating these processes cause mitochondrial diseases, with impaired mitochondrial function and pathological metabolism and signaling [reviewed in 26-27, 37]. The mutation of our patient is located in DNM1Lcausing a disruption in the fission process leads to several functional consequences, expressed in fibroblasts ; including cell growth on glucose-free media, ROS and ATP production and the mitochondrial morphology [10 and present results]. Thus these cells ar suitable as model system for evaluating the effect of small molecules on an individual basis, as we and others have done in the past [38,39]. In the current study, we specifically aimed to evaluate the effect of Bezafibrate, as our preliminary results, screening several small molecules showed promising results (previously unpublished) while other molecules were less effective [40]. To our knowledge, this compound has never been investigated the context of a mitochondrial fission defect. Our result show a positive effect of Bezafibrate on a DNM1L defect with respect to growth on glucose-free medium and a markedly improved ATP production capacity.

This is in accord with previously reports by others including us, showing Bezafibrate to be beneficial for certain mitochondrial diseases. This could possibly be attributed to increased ability to utilize fatty acids $[29,31,32,34,41]$

Furthermore, Bezafibrate had a positive effect on mitochondrial morphology, leading to decreased mitochondrial elongated phenotype, which was approaching controls (higher levels of solitary mitochondria, and lower levels of elongated ones). This might indicate that Bezafibrate has an additional role as a regulator of other factors that participate, directly or indirectly, in other processes such as fission/fusion processes, or mitophagy. Still, the positive effects of Bezafibrate could come with a cost, namely increased ROS production as we observed in this study and our previous findings in some mitochondrial disease cell, albeit the elevations were mild and other cells showed decreased ROS [33,34]. We did not include the evaluation of peroxisomal function in this study since there was no evidence of peroxisomal dysfunction in our patient [10]. To date, few treatments have been suggested for patients suffering from mitochondrial diseases. For the vast majority of patients, the treatment is limited to treating the 
symptoms, rather than curing the disease. Still, the administration of small molecules could improve some mitochondrial parameters, and possibly alleviate mitochondrial disease symptoms [28-32, 42]

Obviously our study is limited to only one patient and was conducted in fibroblasts. Further studies would be needed to show the consistency of the response to Bezafibrate, perhaps using fibroblasts from patients with different mutations in DNM1L. Unfortunately, we were not able to conduct a clinical trial in our patient using Bezafibrate, due to parental noncompliance with the treatment and follow up. However, taking into account the various favorable effects in our study, as well as in other mitochondrial diseases, we suggest that Bezafibrate could be a possible treatment option for patients with certain DNM1L mutations.

\section{Acknowledgements}

This work was supported by a donation from the Marks Family via the Manackerman Charitable Trust UK to A.S.

\section{Author contributions}

Conceptualization, Liza Douiev, Ruth Sheffer, Gabriella Horvath and Ann Saada; Funding acquisition, Ann Saada; Investigation, Liza Douiev and Ann Saada; Methodology, Liza Douiev and Ann Saada; Project administration, Ann Saada; Supervision, Ann Saada; Writing - original draft, Liza Douiev; Writing - review \& editing, Ruth Sheffer, Gabriella Horvath and Ann Saada.

The authors declare no conflicts of interest.

\section{References}

1. DiMauro, S. \& Schon, E. A. Mitochondrial Respiratory-Chain Diseases. 2003, 2656-2668.

2. Twig, G.; Elorza, A.; Molina, A.J.A.; et al. Fission and selective fusion govern mitochondrial segregation and elimination by autophagy. EMBO J. 2008, 27, 433-446.

3. Sauvanet, C.; Duvezin-Caubet, S.; di Rago, J.P; Rojo, M. Energetic requirements and bioenergetic modulation of mitochondrial morphology and dynamics. Semin. Cell. Dev Biol. 2010 , 21(6), 558-65.

4. Westermann, B. Mitochondrial fusion and fission in cell life and death. Nat Rev Mol Cell Biol. 2010, 11, 872-884.

5. Eisner, V.; Picard, M.; Hajnoczky, G. Mitochondrial dynamics in adaptive and maladaptive cellular stress responses. Nature Cell Biology. 2018, 20, 755-765. 
6. Shirihai, O..; Song, M.; Dorn, II G. W. How mitochondrial dynamism orchestrates mitophagy. Circ. Res. 2015, 116(11),1835-1849.

7. Dorn II G. W. Evolving concepts of mitochondrial dynamics. Annu Rev Physiol. 2019, 81, 1-17.

8. Waterham, H.R.; Koster, J.; van Roermund, C.W.T.; et al. A lethal defect of mitochondrial and peroxisomal fission. NEJM. 2007, 356, 1736-1741.

9. Chao, Y. H.; Robak, L. A.; Xia F.; Koenig, M. K.; Adesina, A.; Bacino, C. A.; Scaglia, F.; Bellen H. J.; Wangler M.F. AMissense variants in the middle domain of DNM1L in cases of infantile encephalopaty alter peroxisomes and mitochondria when assayed in Drosphila. Hum Mol Genet. 2016, 25(9), 1846-1856.

10. Sheffer, R.; Douiev, L.; Edvardson, S.; Shaag, A.; Tamimi, K.; Soiferman, D.; Meiner, V. ; Saada A. Postnatal microcephaly and pain insensitivity due to a de novo heterozygous DNM1L mutation causing impaired mitochondrial fission and function. Am J Med Genet A. 2016, 170(6), 1603-1607.

11. Ryan, C.S.; Fine, A.L.; Cohen, A.L.; et al. De novo DNM1L variant in a teenager with progressive paroxysmal dystonia and lethal super-refractory myoclonic status epilepticus. J Child Neurol. 2018, 33, 651-658.

12. Nasca, A.; Legati, A.; Baruffini, E.; et al. Biallelic mutations in DNM1L are associated with a slowly progressive infantile encephalopathy. Hum Mut. 2016, 37, 898-903.

13. Yoon, G.; Boycott, K.M.; et al. Lethal disorder of mitochondrial fission caused by mutations in DNM1L. J Pediatr. 2016, 171, 313-316.

14. Vanstone, J.R.; Smith, A.M.; McBride, S.; et al. DNM1L-related mitochondrial fission defect presenting as refractory epilepsy. Eur J Hum Genet. 2016, 24, 1084-1088.

15. Fahrner, J. A.; Liu, R.; Perry, S. M.; Klein, J. Chan, D.C. A novel de novo dominant negative mutation in DNM1L impairs mitochondrial fission and presents as childhood epileptic encephalopathy. Am J Med Genet A. 2016, 170, 2002-2011.

16. Zaha, K.; Matsumoto, H.; Itoh, M.; et al. DNM1L-related encephalopathy in infancy with Leigh syndrome-like phenotype and suppression-burst. Clin Genet. 2016, 90, 472-474.

17. Hogarth, K.A.; Costford S.R.; Yoon, G. DNM1L variant alters baseline mitochondrial function and response to stress in a patient with severe neurological dysfunction. Biochem Genet. 2018, $56,56-77$.

18. Ladds, E.; Whitney A.; Dombi, E.; et al. De novo DNM1L mutation associated with mitochondrial epilepsy syndrome with fever sensitivity. Neurol Genet. 2018, 4(4), e258.

19. Musto, E.; Gambardella, L.; Contaldo, I.; et al. Epileptic encephalopathy with recurrent focal status epilepticus and epilepsia partialis continua in patient with de novo DNM1L mutation: Electroclinical features. Neuropediatrics. 2018, 49(S01), S1-S12. 
20. Tarailo-Graovac, M.; Zahir, F.R; Zivkovic, I. Genomic analysis of a patient with sensory autonomic neuropathy and severe epileptic encephalopathy reveals a de novo pathogenic DNM1L variant. Molecular Genetics \& Genomic Medicine,2019 accepted

21. Bleazard, W.; McCaffery, J. M.; King, E.J.; Bale S.; Mozdy, A.; Tieu, Q.; Nunnari, J.; Shaw, J.M. The dynamin-related GTPase Dnm1 regulates mitochondrial fission in yeast. Nat Cell Biol. 1999, 1, 298-304.

22. Smirnova, E.; Griparic, L.; Shurland, D. L.; Van Der Bliek, A.M. Dynamin-related protein Drp1 is required for mitochondrial division in mammalian cells. Mol Biol Cell. 2001, 12, 2245-2256.

23. Ingerman, E.; Perkins, E.M.; Marino, M.; et al. Dnm1 forms spirals that are structurally tailored to fit mitochondria. J Cell Biol. 2005, 170, 1021-1027.

24. Chang, C.R. \& Blackstone, C. Dynamic regulation of mitochondrial fission through modification of the dynamin-related protein Drp1. Ann NY Acad Sci. 2010, 1201, 34-9.

25. Friedman, J.R.; Lackner, L.L.; West, M.; DiBenedetto, J.R.; Nunnar,i J.; Voeltz, G.K. ER tubules mark sites of mitochondrial division. Science. 2011, 334, 358-362.

26. Otera, H.; Ishihara, N.; Mihara, K. New insights into the function and regulation of mitochondrial fission. Biochim. Biophys. Acta. 2013, 1833(5):1256-1268.

27. Tilokani, L.; Nagashima, S;. Paupe, V.; Prudent, J.Mitochondrial dynamics: overview of molecular mechanisms. Essays Biochem. 2018,62(3),341-360.

28. Lightowlers, R. H., Taylor, R. H., Turnbull, D. M. Mutations causing mitochondrial disease: What is new and what challenges remain? Science. 2015, 349, 1494-1499.

29. Hirano M.; Emmanuele V.; Quinzii C. M. Emerging therapies for mitochondrial diseases. Essays in Biochemistry. 2018, 62, 467-481

30. Rahman, J; Rahman, S.Mitochondrial medicine in the omics era. Lancet. 2018 391(10139):2560-2574

31. Djouadi, F. \& Bastin, J. Mitochondrial genetic disorders: Cell signaling and pharmacological therapies. Cells. 2019, 8(4), 289.

32. Bonnefont, J.P; Bastin, J.; Behin, A.; Djouadi, F. Bezafibrate for an inborn mitochondrial betaoxidation defect. N. Engl. J. Med. 2009, 360(8),838-340

33. Golubitzky, A.; Dan, P.; Weissman, S.; Link, G.; Wikstrom, J.D.; Saada A. Screening for active small molecules in mitochondrial Complex I deficient patient's fibroblasts, reveals AICAR as the most beneficial compound. PLoS One. 2011, 6(10), e26883.

34. Soiferman, D.; Ayalon, O.; Weissman, S.; Saada, A. The effect of small molecules on nuclearencoded translation diseases. Biochimie. 2014, 100:184-191. 
35. Yu-Wai-Man, P.; Soiferman, D.; Moore, D.G.; Burté, F.; Saada, A. Evaluating the therapeutic potential of idebenone and related quinone analogues in Leber hereditary optic neuropathy. Mitochondrion. 2017, 36, 36-42.

36. Robinson, B. H. Use of fibroblast and lymphoblast cultures for detection of respiratory chain defects. Methods Enzymol. 1996, 264, 454-464.

37. Suomalainen, A. \& Battersby, B.J. Mitochondrial diseases: the contribution of organelle stress responses to pathology. Nat. Rev. Mol. Cell Biol. 2017, 19(2), 77-92.

38. Saada, A. The use of individual patient's fibroblasts in the search for personalized treatment of nuclear encoded OXPHOS diseases. Mol. Genet. Metab. 2011, 104, 39-47.

39. Saada, A. Mitochondria: Mitochondrial OXPHOS (dys) function ex vivo - The use of primary fibroblasts. Int. J. Biochem. Cell Biol. 2014, 48, 60-65.

40. Douiev, L.;Soiferman, D., Alban, C.; Saada, A. The Effects of Ascorbate, N-Acetylcysteine, and Resveratrol on Fibroblasts from Patients with Mitochondrial Disorders. J. Clin. Med. 2016, 6(1). pii: E1. doi: 10.3390/jcm6010001.

41. Waskowicz, L. R.; Zhou, J.; Landau, D. J.; Brooks, E. D.; Lim, A.; Yavarow, Z. A.; Kudo, T.; Zhang, H.; Wu, Y.; Grant, S.; Young, S. P.; Huat B. B.; Yen, P. M.; Koeberl, D. D. Bezafibrate induces autophagy and improves hepatic lipid metabolism in glycogen storage disease type la. Human Molecular Genetics. 2019, 28(1), 143-154.

42. Murphy, M. P. \& Hartley, R. C. Mitochondria as a therapeutic target for common pathologies. Nat. Rev. Drug Discovery. 2018, 17(12), 865-886. 